\title{
The Phenomenon of Life in the Fractal Universe: The Metagalactic Hotbeds of Life Have neither Past nor Future
}

\author{
Sergey Haitun \\ Candidate of Physics and Mathematics, Independent Scholar \\ (Moscow, Russia) \\ E-mail: haitunsd@mail.ru \\ https://orcid.org/0000-0001-5990-7350
}

This article is based on the author's book "The hypothesis of the fractal Universe: Background. Foundations. Twenty-four consequences" (in Russian: Moscow, URSS, 2018, bibl. 428). The cosmic expansion makes it impossible to observe everything beyond the apparent horizon with a radius of about 13.8 billion light-years. Nevertheless, cosmologists quite often transfer the results of observations to the entire Universe, when speaking about the expansion of the Universe, the Big Bang of the Universe, etc. This extrapolation is implicitly based on the (macro)homogeneous Universe hypothesis: in homogeneous Universe its part is in fact similar to the whole. However, the observations of recent years speak of the fractality of the matter distribution in the entire volume of the observable world, which makes the fractal Universe hypothesis more plausible. In such a Universe, a part may differ significantly from the whole. The author includes in the fractal Universe hypothesis the assumption of its infinity, guided by two considerations. Firstly, for the fractal Universe, this assumption is the simplest possible one. Secondly, the infinite fractal Universe has zero global density, which removes the problem of its (Universe) non-stationarity. Since the fractal (and infinite) Universe cannot either expand or contract, the observable expansion is experienced only by our Metagalaxy. Due to the dominance of gravity in metagalaxies with their giant dimensions, they are all non-stationary, constantly experiencing expansion and contraction. With the expansion, metagalaxies evolve, with the contraction, the results of evolution are destroyed. Arising at the regular stage of expansion of the metagalaxy, life each time starts from a blank sheet and disappears without living a trace when it (metagalaxy) contracts.

Keywords: evolution and life, arrangement of fractal Universe, metagalactic hotbeds of life, past and future of life in Universe

Received: January 31, 2019; accepted: April 2, 2019

Philosophy and Cosmology, Volume 23, 2019: 15-21.

https://doi.org/10.29202/phil-cosm/23/2

(C) Haitun, Sergey, 2019 


\section{Introduction}

The results of astronomical observations over the last 10-15 years have made the fractal Universe hypothesis more plausible than the prevailing hypothesis about its (macro) homogeneity. This changes our understanding of the arrangement of the Universe and the phenomenon of life in it. The notions of the role of evolution in the genesis of life remain nevertheless in force.

\section{Evolution and Life}

There is still no agreement in the scientific community regarding the driving force of evolution. Here, the concepts of autogenesis, Darwinism, creationism, etc. compete. The author of these lines adheres to autogenetic views. Here, the driving force of evolution is supposed to be the interactions (matter), which develop (develops) themselves (itself) in the direction of the evolution vector that includes a number of components: increasing complexity and diversity of forms, intensifying interactions, increasing the degree of fractality of evolving systems, etc.

Isaac Newton, Immanuel Kant and Pierre Laplace explained the emergence of complex structures in the Universe by the action of gravity. For instance, Newton, in his letter to Richard Bentley (1692), explained that due to gravity, matter could not remain homogeneously dispersed in space. If space were finite, he explained, and then the substance would gather in one spherical mass. However, in infinite space, matter will gather in an infinite number of spherical masses (stars) [The Correspondence, 1961: 234]. Today we know that under the pressure of gravity, not only stars, but also all sorts of cosmic structures are formed.

I agree, further, with the "Complexity Theory" of the Nobel Prize winner in Physics for 1977, Philip Anderson, who asserts that "at each level of complexity entirely new properties appear" [Anderson, 1972: 393]. At the same time, at different levels of the organization of matter, arising one after another in the course of its (matter) selforganization, all new laws physical, chemical, biological, and social — are applied.

Evolution under the pressure of interactions proceeds the more successfully, the more circumstances allow. This also applies to the phenomenon of life. As Robert Chambers wrote in his book "Vestiges of the Natural History of Creation" (1844), organic "life presses in, as has been remarked, wherever there was room and encouragement for it" [Vestiges, 1863: 110]. For example, from all the planets of the Solar System, life in its developed forms arose only on Earth. On other planets, the pressure of interactions was not as effective.

\section{Invalidity of the theory of natural selection}

The main competitor of the autogenetic theory of evolution today remains the theory of natural selection. Selection in it is only one of the three components of natural selection, which includes:

(1) the emergence of a set of inherited small random (directed "in all directions") mutations;

(2) the survival of the most adaptive of these mutations as a result of the competition of species and their interaction with the environment (that is the selection itself);

(3) the accumulation of small mutations that survive for a number of generations into adaptive traits. 
The second component, which is often incorrectly identified with all natural selection, is quite real, while the first and the third ones do not reflect reality. If the Lord (here Lord is a metaphor) relied only on natural selection, then no evolution would occur.

The first argument. The pace of organic evolution exceeds the pace of evolution of the inorganic environment, so that adaptation to the environment itself could not drive the evolution of the organic world.

Let us clarify this consideration. Life covers the surface of the Earth with a thin layer. Mentally cut a layer of this surface so thick that it includes all living things and all the habitat transformed by it. To do this, it is enough for our surface layer to contain the entire Earth's atmosphere, the entire world ocean and several kilometers of the earth's crust. The rest of the environment, which includes everything inside the Earth, and everything outside the atmosphere, is inorganic. The fact is that the organic world of the Earth - in its broadest sense, evolves much faster than the inorganic environment. This fact, in my opinion, is not only unbreakable, but also inexplicable within the context Darwinism.

The second argument. The adaptability of more and more complex forms, appearing in the course of evolution, often do not exceed the adaptability of the foregoing ones, for instance, bacteria or lichens, showing miracles of survival in the most incredible conditions.

The third argument. In the course of evolutionary changes, given organic species becomes another one, reproductively isolated from the foregoing one, which then often perishes. It is impossible to explain this by adapting to the environment of the foregoing species - why would he want to leave the stage?!

The fourth argument. The positions of the theory of natural selection are also undermined by the evolutionary developmental biology (evo-devo) that has appeared in recent decades. The results obtained here make it possible to assert with more and more confidence that organic evolution happens through macromutations, for the appearance of which there are enough changes in several and even one or two genes.

There are also other arguments against the theory of natural selection being discussed in the literature.

\section{Cosmology has no empirical base}

"I know that I know nothing". These words, usually attributed to Socrates, can be fully referred to our understanding of the Universe. After the discovery of cosmic expansion, it became clear that the observable world is limited for us by the apparent horizon with a radius of about 13.8 billion light-years. Since no signal can propagate faster than light, and the expansion began about 13.8 billion years ago, the events taking place outside this sphere, in principle, cannot be observed by us.

The entire material world, not limited by the apparent horizon, is called the Universe, but its spherical section within the apparent horizon, that is, the world we observe, is called our Metagalaxy. It would be more correctly to call our Metagalaxy a relatively compact cosmic macrostructure, which includes the world we observe and is separated from other metagalaxies in the Universe by distances that are many times larger than its own sizes.

There are no grounds to believe that the sizes of so defined our Metagalaxy coincide with the sizes of the observable world. The radius of the apparent horizon is not determined by the laws of the formation of compact cosmic macrostructures, but by the time elapsed after the onset of the observed Big Bang. The sizes of our Metagalaxy can significantly exceed the sizes of the observable world. 
It follows from the above that cosmology, which studies the Universe as a whole, does not have an empirical base. The rarest or even the only case in the natural sciences. All our statements about the Universe are ineradicably hypothetical. Despite this, cosmologists continually transfer the results of observations to the entire Universe, confidently speaking of the expansion of the Universe, the Big Bang of the Universe, etc. At the same time, they delicately "forget" to report that all this is an extrapolation based on the (macro)homogeneous Universe hypothesis. In such a Universe, a part (our Metagalaxy) is actually similar to the whole (the Universe).

However, the observations of recent years speak for the fractality of the matter distribution in the whole volume of the observable world, which makes the fractal Universe hypothesis more plausible. In such a Universe, a part may differ significantly from the whole.

\section{The fractal Universe hypothesis vs. the (macro)homogeneous Universe hypothesis}

Cosmic structures have a discrete, hierarchically ordered structure, the density of which rapidly decreases with its sizes during the transition from the Sun $\left(1.416 \mathrm{~g} / \mathrm{cm}^{3}\right)$ to our Galaxy $\left(10^{-24} \mathrm{~g} / \mathrm{cm}^{3}\right)$ and the entire observable world $\left(2 \times 10^{-31} \mathrm{~g} / \mathrm{cm}^{3}\right)$. This decrease is described by Edwin Carpenter's well-known empirical law [Carpenter, 1938]: the density of the spherical part of the cosmic structure is proportional to its radius $R$ to a power $(D-3)$, where $D \approx 1,23$. Structures of this kind are now called fractal, and the value $D$ is called their fractal dimension. It is significant that $D$ is less than 3 , that is, the dimension of our three-dimensional space. If $D=3$, the cosmic structures would be homogeneous, i.e. non-fractal.

Ideas about the fractality of the cosmic world contradict the homogeneous Universe hypothesis. To save it, cosmologists have moved to the macrohomogeneous Universe hypothesis, believing that it (Universe) is homogeneous at distances of about or more than 300 million of light-years.

A more precise determination of the upper limit of the distance scales, beyond which the distribution of galaxies is homogeneous required the elaboration of threedimensional maps of the distribution of galaxies supposedly to the greatest depth. This work brought unexpected results: giant cosmic structures were discovered, whose sizes are quite comparable with the radius of the apparent horizon (13.8 billion light-years). We indicate here four such objects with their sizes:

(1) The Sloan Great Wall, about 1.38 billion light-years [Gott et al., 2005].

(2) Huge Large Quasar Group (shining nuclei of galaxies), about $4 \times 2,1 \times 1,2$ billion lightyears [Clowes et al., 2013].

(3) The Hercules-Corona Borealis Great Wall, over 10 billion light-years in one direction and about 7.2 billion light-years in the other direction [Horváth et al., 2015].

(4) The Giant Ring-Like Structure, about 5 billion light-years [Balázs et al., 2015].

After these discoveries, nothing no longer contradicts hypothesis of fractality of the entire observable world, from top to toe. This hypothesis before our very eyes acquires the status of a confirmed empirical fact. Now nothing prevents the extrapolation of this empirical fact to the whole Universe.

Some cosmologists even in these "inhuman" conditions continue to defend the macrohomogeneous Universe hypothesis. They can be understood. In almost all of their theoretical calculations, cosmologists rely not on the equations of the general theory of relativity (GTR) in general form because of their extreme complexity, but on the relatively simple Friedmann equations obtained from them under the assumption of homogeneity of 
the Universe. The rejection of this hypothesis will mean the rejection of these equations. In addition, what then will the cosmologists have?

However, the truth must be looked into the eyes: after the discovery of the giant cosmic structures, the hypothesis of the fractality of the Universe became more plausible than the hypothesis of its macrohomogeneity.

\section{Fractal Universe hypothesis refinement}

Let us call the natural fractals located in our three-dimensional world the ideal ones, if their density is zero. Only the Universe can prove to be such ideal fractal, if it is infinite.

We include in the fractal Universe hypothesis the assumption of its infinity, making it for two reasons. Firstly, for the fractal Universe, this assumption is the simplest possible one. Secondly, Albert Einstein introduced the model of a closed Universe (1917) in order to get rid of its non-stationarity, which arises in the case of a homogeneous Universe with a nonzero density. For a fractal infinite Universe with its zero density, such a problem does not exist.

Briefly, the author's version of the fractal Universe hypothesis can be formulated as follows: the Universe is an ideal fractal.

\section{The arrangement of the fractal Universe}

Guided by the law of parsimony, according to which the simplest of the hypotheses should always be chosen, from the above, we conclude about an extremely simple arrangement of the fractal Universe. There are no artistic frills, such as additional space dimensions, parallel universes, macro-worlds embedded in elementary particles, "wormholes" in space-time, etc.

We have one infinite three-dimensional globally flat space, described by the special theory of relativity (STR). An infinite hierarchically organized set of stars, galaxies, metagalaxies, etc. is dispersed in it. The distances between these objects are many times larger than the sizes of the objects themselves and they grow indefinitely with the sizes of the objects, which ensures such Universe a zero global density.

The fractal Universe is stationary globally, but not locally. The finite-sized macrosystems that compose it can expand and contract as they like, however, these local processes of contraction and expansion throughout the entire Universe cannot prevail over each other. It follows that if the Universe is fractal, then the whole of it did not get through the Big Bang, and the cosmic expansion we observe is the result of the Big Bang only in our Metagalaxy.

Discussing the past of our Metagalaxy, you can rely on the idea of the Big Bounce expressed in the literature regarding the Universe. Apparently, the Big Bang of our Metagalaxy was preceded by its up to the stop contraction, afterwards the gravitational collapse was reversed. Regarding the future of our Metagalaxy the situation is more complicated.

From all the forms of physical interactions, gravitational one is the most longrange. Therefore, it is precisely that it globally dominates in the Universe, as well as in metagalaxies and other sufficiently large cosmic systems. The dominance of gravitational interaction in sufficiently large cosmic systems with nonzero density, as we know, leads to their instability. Only not very large — compared to metagalaxies - cosmic systems, in which other physical interactions are significant, along with gravitational ones, can be in stationary states.

We come to the conclusion that all the metagalaxies dispersed in the Universe (and yet larger systems) are non-stationary due to the dominance of the gravitational interaction in them. Since metagalaxies can only expand and contract without reaching a stationary state, so they do this cyclically. However, due to the irreversibility of these processes, 
the expansion and contraction of metagalaxies are presumably characterized by a kind of residual deformation, which accumulates from cycle to cycle, until one day the metagalaxies interrupt their pulsation, going on to infinite expansion. This, I suppose, is also the future of our Metagalaxy.

Thus, with all its global stationarity, the fractal Universe locally lives a hectic life along its entire space. Its constituent metagalaxies (and yet larger cosmic systems) get through quasicyclic pulsations; all of them have their own duration of life, after which they melt in an infinite expansion, and their contents are gathered up by other metagalaxies or serves as a material for self-organization of new ones.

\section{The evolution stimulation of metagalaxies during their expansion caused by their cooling}

In the course of the expansion of our Metagalaxy after its personal Big Bang, it evolves towards complication. At the stage of contraction, all structures arising during the expansion will be destroyed.

According to the concept of the Hot Big Bang, which is being applied to the Universe today and which we will apply to our Metagalaxy, during the expansion it (our Metagalaxy) has been cooled for about 13.8 billion years. This cooling means a global (within the metagalaxy) transformation of heat (the kinetic energy of randomly moving particles) into other forms of energy. However, energy is a measure of the quantity of interactions. Therefore, the emergence of non-thermal forms of energy means the emergence of forms of interactions that are more ordered than heat.

Since this global process has been going on for billions of years, it stimulates the emergence of more and more complex material structures. One unidirectional process the global evolution of matter towards complication is stimulated by another unidirectional process - the global transformation of heat into other forms of energy.

This can be attributed to all metagalaxies and yet larger cosmic systems: their material contents evolve during the expansion according to all the canons of universal evolution that we mentioned at the beginning of the article. The results of these local evolutions are destroyed during the contraction of these cosmic systems.

Go to the Universe. If it were globally expanding, then global evolution would take place in it towards complication, and if it were contracting, then all structures would be destroyed in it. The impossibility for a fractal Universe of global contraction and expansion means that it does not globally evolve. Finally, how could it globally evolve, if during the cyclic contractions and extensions of its constituent metagalaxies, the results of local evolutions are being vanished each cycle?

\section{Metagalactic hotbeds of life have no past and no future}

As was mentioned above, life arises in the course of evolution wherever conditions allow. There are only eight planets in our Solar System, and a highly organized life has emerged on one of them. In galaxies, there are much more diverse conditions, so the probability of the genesis of life in each of them is substantially greater. Well, in metagalaxies, the probability of the genesis of life, is supposedly altogether close to one.

Arising at the regular stage of expansion of metagalaxy with suitable parameters, life begins with a blank sheet each time, without knowing anything about its predecessors, and disappears without leaving a trace when it (metagalaxy) contracts. In high-temperature 
plasma, into which the contents of metagalaxies transform when they contract, living matter has no chance to survive. Therefore, contrary to Henri Bergson and Vladimir Vernadsky, life arises in the metagalaxies each time completely anew from non-living matter.

Contacts between the hotbeds of life in different metagalaxies are excluded because of the gigantic distances between them (metagalaxies), many times exceeding their own great sizes, which are billions of light-years. And even if some hotbed of life arises in metagalaxy at this stage of its expansion, which ends with the dispersion of the contents of metagalaxy in the intermetagalactic space, then sooner or later it (the contents of the former metagalaxy) will be gathered up by other metagalaxies - already existing or newly formed — and again it will be thrown into the meat grinder of expansions and contractions of their new shelters.

Human individuals are also doomed to death, which does not prevent each of us from living a more or less fulfilling life, filled with joys and sorrows. However, there is a fundamental difference. An individual has a chance to continue to exist in descendants, by making a contribution to the evolution of its society, life on Earth and life in this metagalaxy. The entire hotbed of life in metagalaxy has nothing like this: it (life in metagalaxy) simply closed, leaving no trace behind.

Humanity, probably, will have to accept the ephemeral nature of life, with absence - by cosmological measures - of the past and the future, or ... develop a cosmological hypothesis, more plausible than the fractal Universe hypothesis, which was discussed in this article.

\section{미] References}

Anderson Philip W. More is different. Science, New Series, Volume 177, 1972, Issue 4047, pp. 393-396. https://doi.org/10.1126/science.177.4047.393

Balázs Lajos G., Bagoly Zsolt, Hakkila Jon E., Horváth Istvan, Kóbori József, Rácz Istvan I., Tóth László Viktor. A giant ring-like structure at $0,78<\mathrm{z}<0,86$ displayed by GRBs. Monthly Notices of the Royal Astronomical Society, Volume 452, Issue 3, 2015, pp. 2236-2246. https://doi.org/10.1093/mnras/stv1421

Carpenter Edwin F. Some characteristics of associated galaxies. I. A density restriction in the metagalaxy. Astrophysical Journal, Volume 88, 1938, pp. 344-355. https://doi. org/10.1086/143987

Clowes Roger G., Harris Kathryn A., Raghunathan Srinivasan, Campusano Luis E., Söchting Ilona K., Graham Matthew .J. A structure in the early Universe at z 1.3 that exceeds the homogeneity scale of the R-W concordance cosmology. Monthly Notices of the Royal Astronomical Society, Volume 429, 2013, pp. 2910-2916. https://doi. org $/ 10.1093 / \mathrm{mnras} / \mathrm{sts} 497$

Gott John R. III, Jurić Maro, Schlegel David, Hoyle Fiona, Vogeley Michael, Tegmark Max, Bahcall Neta, Brinkmann Jon. A map of the Universe. The Astrophysical Journal, Volume 624, Issue 2, 2005. pp. 463-484. https://doi.org/10.1086/428890

Haitun, Sergey. The hypothesis of the fractal Universe: Background. Foundations. Twentyfour consequences. Moscow, URSS, 2018. (in Russian).

Horváth Istvan, Bagoly Zsolt., Hakkila Jon., Tóth László Viktor. New data support the existence of the Hercules-Corona Borealis Great Wall. Astronomy and Astrophysisc, Volume 584, 2015, p. A48. DOI: 10.1051/0004-6361/201424829

The Correspondence of Isaac Newton. Cambridge: Cambridge Univ. Press, 1961. Volume 3. - XVIII+445 p.

Vestiges of the Natural History of Creation (Estestvennaya istoriya mirozdaniya). Moskva: Cherpin, Ushakov, 1863. - XIV+366 p. (The anonymous publication, in Russian). 